\title{
Anisotropic friction and excluded volume effects in freely jointed bead-rod polymer chain models
}

\author{
Z. Xu, S. Kim, and J. J. de Pablo \\ Department of Chemical Engineering, University of Wisconsin-Madison, Madison, Wisconsin 53706
}

(Received 10 December 1993; accepted 17 May 1994)

\begin{abstract}
We have studied the kinematics and rheological properties of undiluted polymers by modeling the fluids as a collection of interacting Kramers freely jointed bead-rod chains with anisotropic friction using Brownian dynamics simulations. The anisotropic friction depends on the instantaneous configuration of the system. The resulting stochastic differential equations have multiplicative noise. The correlation of the "Langevin" Brownian forces appearing in these equations turns out to be anisotropic as well. The excluded volume effect, often ignored in kinetic theory, plays an important role in the behavior of short polymer chains. The chain kinematics may be distorted in its absence. In this work, we also discuss the effects of two types of excluded volume (EV) forces (hard-sphere and soft-repulsive forces) on rheological properties of concentrated polymer solutions.
\end{abstract}

\section{INTRODUCTION}

Concentrated polymer solutions and polymer melts represent extremely complex many-particle systems. In these systems, all polymers move simultaneously, and there are too many degrees of freedom to attempt exact analytical descriptions of their dynamics. A widely used approach to handle the kinematics of concentrated polymer solutions and melts relies on the concept of "reptation" originally proposed by de Gennes. ${ }^{1,2}$ In the framework of reptation theories, the many-chain problem is reduced to a much simpler one-chain problem. The approach is based on the introduction of a mean field in which polymer chains diffuse along their backbones. The motion of a macromolecule is confined to a tubelike region which is formed by its neighbors. The first kinetic theory of undiluted polymer solutions and melts based on the reptation picture was developed in a series of papers by Doi and Edwards (DE model) published in 1978 and 1979, ${ }^{3-6}$ and collected in a monograph by Doi and Edwards. ${ }^{7}$ A more refined and very successful phase space theory-developed by Curtiss and Bird ${ }^{8,9}$-made use of a model incorporating the idea of anisotropic hydrodynamic drag forces and Brownian forces. The anisotropic hydrodynamic drag forces express essentially the same idea as the constraining tube in the DE model.

Based on the assumption of reptating motion, Bird and DeAguiar ${ }^{10,11}$ developed the so-called "encapsulated FENE dumbbell" model. With further approximations, they were able to obtain an explicit constitutive equation and the nonlinear rheological properties. A numerical solution of the diffusion equation for this model was completed by Fan. ${ }^{12}$ However, Volkov and Vinogradov ${ }^{13}$ and Phan-Thien and Atkinson $^{14}$ pointed out that for a dilute solution, the fluctuation-dissipation theorem (FDT) gives rise to a relationship between the anisotropy of the hydrodynamic drag forces and the ("Langevin") Brownian forces. While in nonequilibrium melts one does not know to which extent the FDT holds, the expression of Bird et al. ${ }^{15}$ for the anisotropy of the smoothed out Brownian forces (which does not satisfy the FDT) can therefore be regarded as an additional assumption. Baxandall ${ }^{16}$ treated the elastic dumbbell with the same hydrodynamic drag forces as Bird et al ${ }^{15}$ did, but with isotropic Brownian forces (smoothed out). In this paper, we treat polymer chains as freely jointed bead-rod Kramers chains and we discuss the difference between these two types of Brownian forces.

In kinetic theory, excluded volume (EV) effects are often neglected, ${ }^{15}$ this is partly due to difficulties in their mathematical treatment. Recently, Parnas and $\mathrm{Cohen}^{17}$ studied chain conformation with excluded volume effects for freelyjointed bead-rod chain models; the length of their chains, however, was only up to five beads. Fixman's ideas ${ }^{18}$ for treating a model with hydrodynamic interaction (HI) and EV have also been revived; ${ }^{19}$ based on both a transformation to the normal modes and a boson operator formulation, Ahn et al. ${ }^{19}$ were able to solve a long-chain problem without much computational effort. However, the ideal tool for testing EV effect is Brownian dynamics simulation since no approximations are required.

The organization of this paper is as follows. In Sec. II the theoretical basis is given. We begin by developing the stochastic differential equations and the molecular stress tensor for a freely jointed bead-rod chain with anisotropic friction. Our development follows that of $\mathrm{Liu}^{20,21}$ for Kramers chains (no HI and no EV). In Sec. III, we present the details of the Brownian dynamics simulation technique and the solution of the corresponding set of stochastic differential equations (SDE). The purpose of our work is the determination of nonlinear rheological properties in steady flow fields. Section IV presents the results for different flow fields and a brief comparison to experiment. Section V concludes the paper.

\section{THEORY}

Here we study a freely jointed bead-rod chain model in which the polymer is modeled as a series of beads joined by freely rotating rods. Before presenting the formal derivation of the corresponding stochastic differential equations, we adopt the following conventions for indices: $\mu, \nu, \eta, \ldots$ to designate beads $1,2, \ldots, N ; i, j, k, \ldots$ to designate rods of the chain $1,2, \ldots, N-1 ; p, a, r, \ldots$ to designate orientation of a rod 1.2. 
The ranges of the summation indices, unless stated otherwise, will be implied by this convention. Superscripts $(h)$, $(B),(c)$, and (EV) denote hydrodynamic force, Brownian force, constraining force, and excluded volume force, respectively. Boldface characters (both Greek letters and Roman letters) indicate that a quantity is a vector or a tensor. The following assumptions, which are commonly used in kinetic theory, ${ }^{15}$ are employed in this work: The polymer chain is imbedded in a mean field in which the beads of the chain are subject to two types of forces from the neighboring chains. One is the deterministic hydrodynamic (or drag) force, which is an anisotropic modification of Stokes' law. The other is the random force with which the other polymer molecules incessantly bombard the acting polymer molecule. The difference between the hydrodynamic forces on adjacent pairs of beads is assumed to depend not only on the difference of velocities but also on the orientation of the rod, i.e.,

$$
\mathbf{F}_{k+1}^{(h)}-\mathbf{F}_{k}^{(h)}=-\zeta_{k} \cdot\left(\dot{\mathbf{r}}_{k+1}-\dot{\mathbf{r}}_{k}\right),
$$

where the friction tensor $\zeta_{k}$ is

$$
\zeta_{k}=\zeta\left[\delta-(1-\epsilon) \mathbf{u}_{k} \mathbf{u}_{k}\right] .
$$

Here $\dot{\mathbf{r}}_{k}$ is the velocity of the $k$ th bead (we are forced to use this inconsistent notation for beads in order to match the convention for links), $\mathbf{u}_{k}$ is the $k$ th link vector, and $\delta$ designates the unit tensor. Two empirical constants appear in this modified Stokes' law expression; the scalar friction coefficient $\zeta$ and the link tension coefficient $\epsilon$. Parameter $\epsilon$, which determines the anisotropy of the friction tensor, is taken here to be a constant, although it could also be taken to be a function of shear rate. If $\epsilon=1$, the friction tensor $\zeta_{k}$ is isotropic and we recover the simple Stokes' law. If $\epsilon=0$, there is no component of the difference of the forces on two succeding beads in the direction of the link joining them. It is experimentally found that the range of $\epsilon$ is from 0.3 to 0.5 for concentrated solutions and undiluted polymers, both monodisperse and polydisperse. ${ }^{22,23}$ The proposed expression for $\zeta_{k}$ is based on the concept that hydrodynamic forces are larger along the backbone (link) than in the direction perpendicular to the backbone. These forces therefore enhance the motion of the polymer chain along the direction of its backbone or hinder motion in directions perpendicular to the backbone.

The random Brownian forces are assumed to follow a behavior similar to that of the hydrodynamic forces [Eqs. (1) $-(2)$ ], which depend on the orientation of the rod, i.e.,

$$
\mathbf{F}_{k+1}^{(B)}-\mathbf{F}_{k}^{(B)}=\alpha \Omega_{k}^{1 / 2} \cdot\left(\mathbf{f}_{k+1}-\mathbf{f}_{k}\right) .
$$

Here $\mathbf{f}_{k}$ is a Gaussian white noise acting on the $k$ th bead. Parameter $\alpha$ is a constant with the value $\sqrt{2 k T \zeta}$. The tensors $\Omega_{k}$ are

$$
\Omega_{k}=\delta-(1-\epsilon) \mathbf{u}_{k} \mathbf{u}_{k} .
$$

Note that by construction this choice of $\Omega_{k}$ satisfies the fluctuation-dissipation theorem at equilibrium. The form of the Brownian forces is different from that used in the DE and $\mathrm{CB}$ models. These Brownian forces, together with anisotropic hydrodynamic forces, confine the polymer chains to a tubelike region having a variable diameter.
The momenta attain equilibrium much faster than the positions of the beads. This implies that the inertial terms appearing in the equation of motion for the beads can be assumed to be negligible. ${ }^{24}$

The flow field is assumed to be homogeneous, so that the velocity of the solution at the position of bead $\nu$ can always be written as

$$
\mathbf{v}\left(\mathbf{r}_{\nu}\right)=\mathbf{v}_{0}+\left[\kappa(t) \cdot \mathbf{r}_{\nu}\right]
$$

where $\mathbf{v}_{0}$ is independent of the position of the fluid element, $\mathbf{r}_{\nu}$ is the location of bead $\nu$, and $\kappa$ is the transpose of the velocity gradient tensor. The tensor $\kappa$ is independent of position but may be a function of time $t$; it is also traceless because the fluid is assumed to be incompressible.

\section{A. Stochastic differential equations}

With those assumptions, we can write out the Langevin equation for the $\nu$ th bead in the bead-rod Kramers chain

$$
\mathbf{F}_{\nu}^{(h)}+\mathbf{F}_{\nu}^{(B)}+\mathbf{F}_{\nu}^{(c)}+\mathbf{F}_{\nu}^{(\mathrm{EV})}=0,
$$

The last term $\mathbf{F}_{\nu}^{(\mathrm{EV})}$ expresses the intramolccular bead-bead interaction due to excluded volume effects. Rather than complicating the problem with EV forces, we present the derivation of the stochastic differential equations (SDE) for phantom chains; we discuss EV forces at the end of this section and leave the derivation of the SDE including EV forces (incorporated by using a soft repulsive potential) to Appen$\operatorname{dix}$ A.

The hydrodynamic force $\mathbf{F}_{\nu}^{(h)}$ acting on bead $\nu$ can be shown to be $\mathrm{e}^{15}$

$$
\mathbf{F}_{\nu}^{(h)}=-\sum_{\mu} \tilde{\zeta}_{\nu \mu} \cdot\left[\dot{\mathbf{r}}_{\mu}-\mathbf{v}\left(\mathbf{r}_{\mu}\right)\right]+\frac{1}{N} \mathbf{F}^{(h)},
$$

where

$$
\tilde{\zeta}_{\nu \mu}=\sum_{k} B_{\nu k} \zeta_{k} \bar{B}_{k \mu}
$$

The total hydrodynamic force [see Eqs. 18.2-16, Ref. 15] on a macromolecule is

$$
\mathbf{F}^{(h)}=\sum_{\nu} \mathbf{F}_{\nu}^{(h)}
$$

Since Eq. (1) only defines a hydrodynamic force difference, the total force $\mathbf{F}^{(h)}$ has not been specified yet. In this development, we take the total hydrodynamic force on a macromolecule to be

$$
\mathbf{F}^{(h)}=-\zeta^{(t)}\left[\dot{\mathbf{r}}_{c}-\mathbf{v}\left(\mathbf{r}_{c}\right)\right],
$$

where $\zeta^{(t)}=N \zeta$ is the scalar friction coefficient of the polymer molecule as a whole. Equation (10) constitutes perhaps the most severe approximation so far; we will discuss it again later. The matrix elements $B_{\nu k}$ and $\bar{B}_{k \mu}$ in Eq. (8) are defined by,

$$
\begin{aligned}
& B_{\nu k}=\left\{\begin{array}{l}
k / N \quad k<\nu \\
-[1-(k / N)] \quad k \geqslant \nu
\end{array}\right. \\
& \bar{B}_{k \nu}=\delta_{k+1, \nu}-\delta_{k, \nu} .
\end{aligned}
$$


Similarly, the Brownian forces $\mathbf{F}_{v}^{(B)}$ can be shown to be of the form ${ }^{15}$

$$
\mathbf{F}_{\nu}^{(B)}=\alpha \sum_{\mu} \tilde{\Omega}_{\nu \mu} \cdot \mathbf{f}_{\mu}+\frac{1}{N} \mathbf{F}^{(B)},
$$

where

$$
\tilde{\Omega}_{\nu \mu}=\sum_{k} B_{\nu k} \Omega_{k}^{1 / 2} \vec{B}_{k \mu},
$$

and where

$$
\mathbf{F}^{(B)}=\sum_{\nu} \mathbf{F}_{\nu}^{(B)}
$$

is the total random force acting on a polymer molecule. The $\mathbf{f}_{\mu}$ appearing in Eq. (13) denotes the Gaussian white noise acting on the $\mu$ th bead. According to the definition of total hydrodynamic force on the polymer molecule [Eq. (10)], we can obtain the $\mathbf{F}^{(B)}$ from the fluctuation-dissipation theorem at equilibrium

$$
\mathbf{F}^{(B)}=\sqrt{2 k T \zeta^{(t)}} \mathbf{f}
$$

where $f$ represents the total white noise acting on the whole polymer chain, $\mathbf{f}=\Sigma_{\mu} \mathbf{f}_{\mu}$.

The constraint forces $F_{\nu}^{(c)}$ acting on the $\nu$ th bead have been discussed by $\mathrm{Liu}^{20.21}$ and are given by

$$
\mathbf{F}_{\nu}^{(c)}=-\sum_{i} \gamma_{i} \frac{\partial}{\partial \mathbf{r}_{\nu}} \sigma_{i}=-2 \sum_{i} \gamma_{i} \bar{B}_{i \nu}\left(\mathbf{r}_{i+1}-\mathbf{r}_{i}\right),
$$

where the $\gamma_{i}$ are $N-1$ undetermined Lagrange multipliers associated with $N-1$ holonomic constraints given by

$$
\sigma_{i}=\left(\mathbf{r}_{i+1}-\mathbf{r}_{i}\right)^{2}-a^{2}=0 .
$$

Here $r_{i}$ denotes the location of the $i$ th bead. Constant $a$ is the separation between two successive beads (i.e., the bond length).

The next step in our development is to obtain a stochastic differential equation for the velocity $\dot{\mathbf{u}}_{j}$ which, when introduced into the equation of continuity, leads to a FokkerPlanck equation for the distribution function. To find an expression for $\dot{\mathbf{u}}_{j}$, we first insert Eqs. (7), (13), and (17) into Eq. (6), and obtain

$$
\dot{\mathbf{r}}_{\nu}=\kappa \cdot \mathbf{r}_{\nu}+\alpha \sum_{\mu} \mathbf{H}_{\nu \mu} \cdot \mathbf{f}_{\mu}+\sum_{\mu} \tilde{\zeta}_{\nu \mu}^{-1} \cdot \mathbf{F}_{\mu}^{(c)},
$$

where the tensor $\mathbf{H}_{\nu \mu}$ is given by $\Sigma_{\eta} \tilde{\zeta}_{\nu \eta}^{-1} \cdot \tilde{\Omega}_{\eta \mu}$. Subtracting the equation for the $j$ th bead from that for the $(j+1)$ th bead we get

$$
\begin{aligned}
\dot{\mathbf{u}}_{j}= & \boldsymbol{\kappa} \cdot \mathbf{u}_{j}+\frac{\alpha}{a} \sum_{\nu} \sum_{\mu} \bar{B}_{j \nu} \mathbf{H}_{\nu \mu} \cdot \mathbf{f}_{\mu} \\
& -2 \sum_{i} \gamma_{i} \sum_{\mu, \nu} \bar{B}_{j \nu} \bar{\zeta}_{\nu \mu}^{-1} \bar{B}_{i \mu} \cdot \mathbf{u}_{i} .
\end{aligned}
$$

In deriving Eq. (20), we used the fact that the $\mathbf{F}^{(h)}$ and $\mathbf{F}^{(B)}$ cancel each other by summing over all beads in the equation of motion and the fact that the summation of constraint forces vanishes for a whole polymer molecule.

In order to obtain the Fokker-Planck equation, we have to determine the Lagrange multipliers $\gamma_{i}$. To solve for $\gamma_{i}$, we recall that the length of the rods is fixed. We therefore set the derivative of Eq. (18) with respect to time $t$ to be zero,

$$
\frac{d}{d t}\left(\mathbf{u}_{j}^{2}-1\right)=2 \mathbf{u}_{j} \cdot \dot{\mathbf{u}}_{j}=0 .
$$

Inserting Eq. (20) into Eq. (21), we obtain

$$
\gamma_{i}=\frac{1}{2} \sum_{k} \hat{C}_{i k}\left(\kappa: \mathbf{u}_{k} \mathbf{u}_{k}+\frac{\alpha}{a} \sum_{\mu} \sum_{\nu} \bar{B}_{k \nu} \mathbf{H}_{\nu \mu}: \mathbf{f}_{\mu} \mathbf{u}_{k}\right),
$$

where $\hat{C}_{i k}$ are the elements of the matrix inverse to $\hat{A}_{j i}$. The matrix $\hat{A}_{j i}$ is given by

$$
\hat{A}_{j i}=\sum_{\mu} \sum_{\nu} \bar{B}_{j \nu} \tilde{\zeta}_{\nu \mu}^{-1} \bar{B}_{i \mu}: \mathbf{u}_{i} \mathbf{u}_{j}
$$

Inserting the formal expression for $\gamma_{i}$ given in Eq. (22) into Eq. (20), we obtain

$$
\begin{aligned}
\dot{\mathbf{u}}_{j}= & \kappa \cdot \mathbf{u}_{j}-\sum_{i k} \mathbf{A}_{j i} \hat{C}_{i k}\left(\kappa: \mathbf{u}_{k} \mathbf{u}_{k}\right) \cdot \mathbf{u}_{i}+\frac{\alpha}{a} \sum_{\mu}\left[\sum_{\nu} \vec{B}_{j \nu} \mathbf{H}_{\nu \mu}\right. \\
& \left.-\sum_{i k} \hat{C}_{i k} \bar{B}_{k \nu}\left(\mathbf{A}_{j i} \cdot \mathbf{u}_{i}\right) \mathbf{u}_{k} \cdot \mathbf{H}_{\nu \mu}\right] \cdot \mathbf{f}_{\mu} \\
= & \sum_{i k} \mathbf{A}_{j i} \cdot \mathbf{K}_{i k} \cdot \kappa \cdot \mathbf{u}_{k}+\frac{\alpha}{a} \sum_{\mu \nu} \sum_{i k} \mathbf{A}_{j i} \cdot \mathbf{K}_{i k} \cdot \mathbf{H}_{\nu \mu} \ddot{B}_{k \nu} \cdot \mathbf{f}_{\mu},
\end{aligned}
$$

where the second-rank tensor $\mathbf{K}_{i k}$ is defined by

$$
\mathbf{K}_{i k}=\mathbf{C}_{i k}-\hat{C}_{i k} \mathbf{u}_{i} \mathbf{u}_{k} .
$$

The matrix $\mathbf{C}_{i k}$ is the inverse of the matrix $\mathbf{A}_{j i}$. The matrix $\mathbf{A}_{j i}$ is defined by

$$
\mathbf{A}_{j i}=\sum_{\mu} \sum_{\nu} \bar{B}_{j \nu} \tilde{\zeta}_{\nu \mu}^{-1} \bar{B}_{i \mu}
$$

Equation (24) forms a set of Stratonovich stochastic differential equations describing sample trajectories for a single Kramers chain in a concentrated polymer solution.

We can now discuss the inclusion of EV forces into Eq. (6). In explicit molecular dynamics or Monte Carlo simulations, the interaction between particles are approximated via a variety of potential-energy functions [e.g., a Lennard-Jones (LJ) potential]. In Brownian dynamics, the choice of function is not clear because each bead represents many monomers of the polymer chain. Purely repulsive forces have been applied in most Brownian dynamics simulations. ${ }^{17}$ For simplicity, in most of our calculations we will treat EV interactions by virtue of a hard sphere (HS) potential or a purely repulsive soft potential. For the HS potential we use

$$
\phi_{i j}= \begin{cases}0 & r_{i j}>d \\ \infty & r_{i j} \leqslant d\end{cases}
$$


where $r_{i j}$ is the distance separating the centers of the beads $i$ and $j$, and $d$ is the diameter of the bead. For the soft potential we use

$$
\phi_{i j}=\left\{\begin{array}{l}
0 \quad r_{i j}>d \\
\frac{4 C}{k T}\left(\frac{a}{r_{i j}}\right)^{m} \quad r_{i j} \leqslant d
\end{array}\right.
$$

Constant $C$ has units of energy, $k$ is Boltzmann's constant, and $T$ is the temperature. When exponent $m$ is equal to 12 , this interaction force corresponds to the repulsive part of $\mathrm{LJ}$ potential.

\section{B. Fokker-Planck equation}

To describe a stochastic process, one can use either a set of stochastic differential equations or the equivalent FokkerPlanck equation. In general, it is convenient to choose a set of generalized coordinates to write the Fokker-Planck equation. For the Kramers chain model, one often selects a set of polar coordinates $Q_{k 1}=\theta_{k}, Q_{k 2}=\phi_{k}, k=1,2, \ldots, N-1$ describing the orientation of the unit vector $\mathbf{u}_{k}$. We can then obtain the Stratonovich stochastic differential equation for the generalized coordinates $Q_{i p}$ from Eq. (24),

$$
\begin{aligned}
\dot{Q}_{i p}= & \mathbf{d}_{i p} \cdot \sum_{j k} \mathbf{A}_{i j} \cdot \mathbf{K}_{j k} \cdot \kappa \cdot \mathbf{u}_{k}+\frac{1}{a} \sqrt{\frac{2 k T}{\zeta}} \mathbf{d}_{i p} \\
& \cdot \sum_{\mu} \sum_{j k} \mathbf{A}_{i j} \cdot \mathbf{K}_{j k} \cdot \sum_{\nu} \mathbf{H}_{\nu \mu} \bar{B}_{k \nu} \cdot \mathbf{f}_{\mu} .
\end{aligned}
$$

In deriving this last equation we used the relation

$$
\dot{\mathbf{u}}_{i}=\sum_{p} \mathbf{c}_{i p} \dot{Q}_{i p},
$$

where the $\mathbf{d}_{i p}$ are the reciprocal base vectors to spherical base unit vectors $\mathbf{c}_{i p}{ }^{15}$

These stochastic differential equations can also be rewritten as

$$
\dot{Q}_{i p}=h_{i p}+\sum_{\mu} \beta_{i p, \mu} \cdot \mathbf{f}_{\mu} .
$$

Equating Eqs. (29) and (31) we see that

$$
\begin{aligned}
& h_{i p}=\mathbf{d}_{i p} \cdot \sum_{j k} \mathbf{A}_{i j} \cdot \mathbf{K}_{j k} \cdot \boldsymbol{\kappa} \cdot \mathbf{u}_{k}, \\
& \beta_{i p, \mu}=\frac{1}{a} \sqrt{\frac{2 k T}{\zeta}} \mathbf{d}_{i p} \cdot \sum_{j k} \mathbf{A}_{i j} \cdot \mathbf{K}_{j k} \cdot \sum_{\nu} \mathbf{H}_{\nu \mu} \bar{B}_{k \nu} .
\end{aligned}
$$

The Fokker-Planck equation equivalent to the Stratonovich stochastic differential Eq. (24) can now be formulated as

$$
\begin{aligned}
\frac{\partial}{\partial t} \psi(Q, t)= & -\sum_{i p} \frac{\partial}{\partial Q_{i p}} h_{i p} \psi+\frac{1}{2} \sum_{i j p q} \sum_{\mu} \frac{\partial}{\partial Q_{i p}} \beta_{i p, \mu} \\
& \cdot \frac{\partial}{\partial Q_{j q}} \beta_{j q, \mu} \psi
\end{aligned}
$$

$$
\begin{aligned}
= & -\sum_{i p}\left[\mathbf{d}_{i p} \cdot \sum_{j k} \mathbf{A}_{i j} \cdot \mathbf{K}_{j k} \cdot \kappa \cdot \mathbf{u}_{k}\right] \psi+\frac{k T}{\zeta a^{2}} \\
& \times \sum_{i j k l_{p q}} \sum_{\mu} \frac{\partial}{\partial Q_{i p}}\left(\mathbf{d}_{i p} \cdot \mathbf{A}_{i k} \cdot \mathbf{K}_{k l} \cdot \sum_{\nu} \mathbf{H}_{\nu \mu} \bar{B}_{l \nu}\right) \\
& \cdot \frac{\partial}{\partial Q_{j q}}\left(\mathbf{d}_{j q} \cdot \sum_{m n} \mathbf{A}_{j m} \mathbf{K}_{m n} \cdot \sum_{\eta} \mathbf{H}_{\eta \mu} \bar{B}_{n \eta}\right) \psi
\end{aligned}
$$

This is the first time that the Fokker-Planck equation for anisotropic Langevin Brownian forces has been given. Note that the complexity of this equation makes difficult its analytical solution.

\section{Stress tensors}

Our starting point for derivation of an expression for the stress tensor is the general expression [Eq. 18.1-10] given in Ref. 15,

$$
\tau=-n N \sum_{\nu}\left\langle\mathbf{R}_{\nu} \mathbf{F}_{\nu}^{(h)}\right\rangle,
$$

where $n$ is the number density of the polymer chains. Vector $\mathbf{R}_{\nu}$ denotes the position of bead $\nu$ relative to the center of mass of the chain. $N$ is the number of beads in a chain molecule. It has the following relation to the link vectors:

$$
\mathbf{R}_{\nu}=a \sum_{i} B_{\nu i} \mathbf{u}_{i}
$$

Substituting Eqs. (7) and (36) into Eq. (35), we get

$$
\tau=n N \sum_{\nu}\left\langle\mathbf{R}_{\nu}\left[\sum_{\mu} \sum_{k} B_{\nu k} \zeta_{k} \bar{B}_{k \mu} \cdot\left(\dot{\mathbf{r}}_{\mu}-\kappa \cdot \mathbf{r}_{\mu}\right)-\frac{1}{N} \mathbf{F}^{(h)}\right]\right\rangle .
$$

The second term in the above equation is zero since $\Sigma_{\nu} \mathbf{R}_{\nu}=0$. Inserting the expression for $\zeta_{k}[\mathrm{Eq}$. (2)] into Eq. (37) yields

$$
\tau=n N \zeta a^{2} \sum_{i k} C_{i k}\left\langle\mathbf{u}_{i}\left[\dot{\mathbf{u}}_{k}-\kappa \cdot \mathbf{u}_{k}+(1-\epsilon) \kappa: \mathbf{u}_{k} \mathbf{u}_{k} \mathbf{u}_{k}\right]\right\rangle,
$$

where $C_{i k}$ is the Kramers matrix, i.e.,

$$
C_{i k}=\sum_{\nu} B_{\nu i} B_{\nu k}
$$

From Eq. (38), we find that the symmetric part of the stress tensor is

$$
\begin{aligned}
\tau= & \frac{1}{2} n N \zeta a^{2} \sum_{i k} C_{i k}\left[\frac{\partial}{\partial t}\left\langle\mathbf{u}_{i} \mathbf{u}_{k}\right\rangle-\left\langle\mathbf{u}_{i} \mathbf{u}_{k}\right\rangle \cdot \kappa^{\dagger}-\kappa \cdot\left\langle\mathbf{u}_{i} \mathbf{u}_{k}\right\rangle\right. \\
& \left.+(1-\epsilon)\left\langle\left(\mathbf{u}_{i} \mathbf{u}_{k}+\mathbf{u}_{k} \mathbf{u}_{i}\right) \mathbf{u}_{k} \mathbf{u}_{k}\right\rangle: \kappa\right]
\end{aligned}
$$


where superscript $\dagger$ denotes a transpose. This expression is very similar to Eq. (19.4-4) (Ref. 15) as derived from phasespace kinetic theory.

\section{COMPUTER SIMULATION}

Solving the high-dimensional Fokker-Planck cquation (34) for the probability distribution function $\psi$ either analytically or numerically is difficult. An alternative but equally valid approach is to perform a Brownian dynamics simulation of the corresponding Langevin equations (24), which are exactly equivalent to the Fokker-Planck equation (34). The presence of constraints, however, presents some difficulties; the constraints are satisfied exactly only at some initial time, and as time proceeds in the numerical integration of a large set of coupled differential equations, the computed trajectories deviate more and more from the exact ones. In order to avoid these difficulties, Ryckaert et al. ${ }^{25}$ developed the socalled SHAKE algorithm. This algorithm first treats the system as if it were unconstrained. It then uses an iterative correction to arrive at the constrained results. The next step is to apply the SHAKE idea to our work, and develop an expression suitable for numerical treatment.

We can rewrite the displacement of a bead that occurs in a finite time step $\Delta t$ as a sum of two terms,

$$
\mathbf{r}_{\nu}(t+\Delta t)=\mathbf{r}_{v}^{\prime}(t+\Delta t)+\delta \mathbf{r}_{\nu}
$$

The first term $\mathbf{r}_{\nu}^{\prime}(t+\Delta t)$ is the position which would have been reached in the absence of constraints. The second one, $\delta \mathbf{r}_{\nu}$, is the correction to $\mathbf{r}_{\nu}^{\prime}(t+\Delta t)$ due to the constraint forces. The bead position after an unconstrained move, $\mathbf{r}_{\nu}^{\prime}(t+\Delta t)$, is calculated in a straightforward manner

$$
\mathbf{r}_{\nu}^{\prime}(t+\Delta t)=\mathbf{r}_{\nu}(t)+\left[\kappa \cdot \mathbf{r}_{\nu}(t)\right] \Delta t+\alpha \sum_{\mu} \check{\zeta}_{\nu \mu}^{-1} \cdot \Delta \mathbf{W}_{\mu}^{\prime},
$$

where $\Delta \mathbf{W}_{\mu}^{\prime}$ is

$$
\Delta \mathbf{W}_{\mu}^{\prime}=\sum_{\eta} \sum_{k} B_{\mu k} \Omega_{k}^{1 / 2} \bar{B}_{k \eta} \cdot \Delta \mathbf{W}_{\eta} .
$$

The Wiener processes $\Delta \mathbf{W}_{\eta}$ are chosen from a Gaussian distribution characterized by its first and second moments,

$$
\left\langle\Delta \mathbf{W}_{\nu}(t)\right\rangle=0 \text {, }
$$

$$
\left\langle\Delta \mathbf{W}_{\nu}(t) \Delta \mathbf{W}_{\mu}(t)\right\rangle=\delta_{\nu \mu} \delta \Delta t .
$$

The second term in Eq. (41), $\delta r_{\nu}$, is given by

$$
\delta \mathbf{r}_{\nu}=-\frac{2 \Delta t}{\zeta} a \sum_{i} \sum_{\mu} \zeta \gamma_{i} \tilde{\zeta}_{\nu \mu}^{-1} \bar{B}_{i \mu} \cdot \mathbf{u}_{i},
$$

where the Lagrange multipliers are unknown. In the SHAKE algorithm, the $\gamma_{i}$ are determined so that the constraint equations

$$
\sigma_{i}=\left[\mathbf{u}_{i}(t+\Delta t)\right]^{2}-1=0
$$

are satisfied within a specified tolerance at each time step.

Inserting Eqs. (41), (42), and (46) into the constraint Eq.

(47) leads to a set of quadratic equations for $\gamma_{i}$,

$$
\begin{aligned}
& 2 \sum_{i} \gamma_{i}^{\star(n)} \sum_{\mu \nu} \zeta \bar{B}_{i \mu} \tilde{\zeta}_{\nu \mu}^{-1}: \mathbf{u}_{i}(t) \mathbf{u}_{j}^{\prime}(t+\Delta t) \bar{B}_{j \nu} \\
&=\left[\mathbf{u}_{j}^{\prime 2}(t+\Delta t)-1\right]+\left[\sum_{i} \gamma_{i}^{\star(n-1)} \sum_{\mu \nu} \zeta \bar{B}_{i \mu} \tilde{\zeta}_{\nu \mu}^{-1}\right. \\
&\left.\cdot \mathbf{u}_{i} \bar{B}_{j \nu}\right]^{2},
\end{aligned}
$$

where $\gamma_{i}^{*}=(2 \Delta t / \zeta) \gamma_{i}$. The Lagrange multipliers $\gamma_{i}^{\star}$ can be obtained by iterating with initial $\gamma_{i}^{*(0)}=0$ until all the constraints are satisfied within a specified tolerance. Convergence is usually rapid, but the method is slow when the system of equations is large. Once all the constraints are satisfied within the specified tolerance, the corrections $\delta \mathbf{r}_{\nu}$ are calculated via Eq. (46), and the new bead position $\mathbf{r}_{\nu}(t+\Delta t)$ is determined from Eq. (41).

In statistical mechanics, the rheological and thermodynamic properties of the system are obtained by averaging over ensembles. In this investigation, we are interested only in stationary properties, so we invoke the principle of ergodicity and work with time averages over a single trajectory instead of ensemble averages. To minimize finite time step effects we perform different runs for different time steps and then extrapolate to $\Delta t=0$. We find that the error due to finite time step is negligible when the conditions $\Delta t / \lambda<10^{-3}$ and $\dot{\gamma} \Delta t<10^{-3}$ are satisfied. We observe this condition for $\Delta t$ in all of our simulations.

In this work we treat intramolecular bead-bead interactions by a HS or by a soft repulsive potential-energy function. Since a HS potential produces an infinite force when two beads are in contact with each other, we cannot explicitly take this force into account in our SDE. The way we handle this excluded volume effect for hard sphere interactions is by simulating phantom chains; we then verify that the distances between beads are greater than the bead diameter, at every time step. If an overlap is produced, the time step is reduced until the overlap is eliminated. Note, however, that if the time step has to be reduced below a certain limit, for example, $2^{-3} \Delta t$, a different set of Brownian forces is generated.

A better understanding of the effects of EV on chain dynamics and rheological properties can be gained by employing a soft repulsive potential (e.g., Lennard-Jones); by means of a soft potential, we can explicitly include EV forces in the SDE as well as in the stress tensor, thereby quantifying their effects on the behavior of the system. Moreover, a soft potential provides a more realistic description of molecular forces than a simple HS potential. The required SDEs for chains interacting through a soft repulsive potential are derived in Appendix A. The results of simulations using a soft potential are compared to those obtained for a hard sphere potential in the next section.

\section{RESULTS AND DISCUSSION}

The set of stochastic differential equations (19) is of the Stratonovich-type. Stratonovich integration in computer simulation is difficult because it requires information at the 


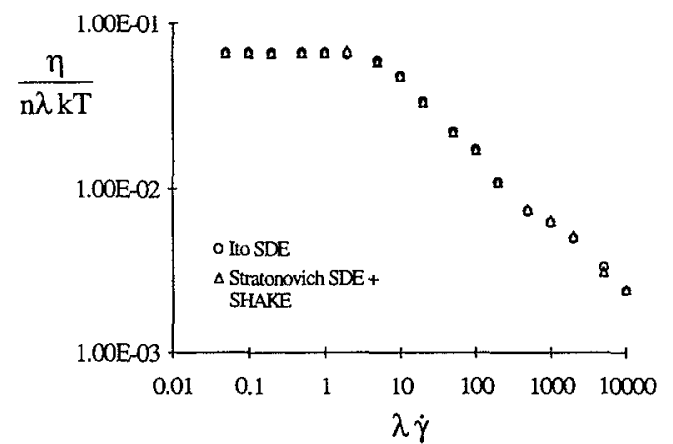

FIG. 1. Comparison of reduced steady shear viscosity for rigid dumbbells from Brownian dynamics simulations for Ito SDE (circle), and Stratonovich SDE with SHAKE (triangle).

current time and at the next future time for which we are trying to solve the equations. However, $\mathrm{Liu}^{20,21}$ has shown that the trajectories constructed from a stratonovich SDE combined with the SHAKE algorithm locally converge to those from the corresponding Ito SDE in the mean square sense (for the Kramers chain with isotropic hydrodynamic forces). For chains of up to three beads, we have also been able to derive the explicit Ito SDE. Unfortunately, we have been unable to derive corresponding equations for an arbitrary number of beads in our model. We have shown numerically that the trajectories generated by the algorithm described in this paper for rigid dumbbells locally converge to those from an Ito SDE. For rigid dumbbells, the results of Brownian dynamics simulations from both the Ito SDE and the Stratonovich SDE + SHAKE algorithms for steady shear flow are shown in Figs. 1-3. Figure 1 shows the viscosity function against shear rate. It can be seen that both approaches exhibit identical shear-thinning behavior. Figures 2 and 3 show the first and second normal stress differences vs shear rate. All computed points show that the difference between these two approaches is negligible. We conclude that, for dumbbells, the trajectories generated by our Stratonovich SDE+SHAKE algorithm converge to those generated by the Ito SDE. Since the mathematical difficulties prohibit treatment of a model with more than three beads, we simply

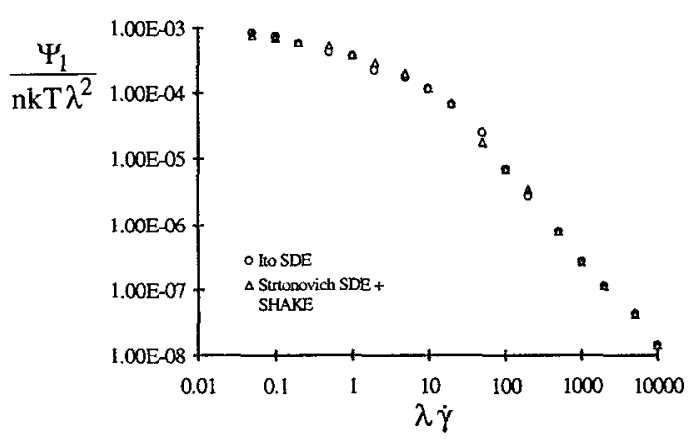

FIG. 2, Comparison of reduced first normal stress difference for rigid dumbbells from Brownian dynamics simulations for Ito SDE (circle), and Stratonovich SDE with SHAKE (triangle).

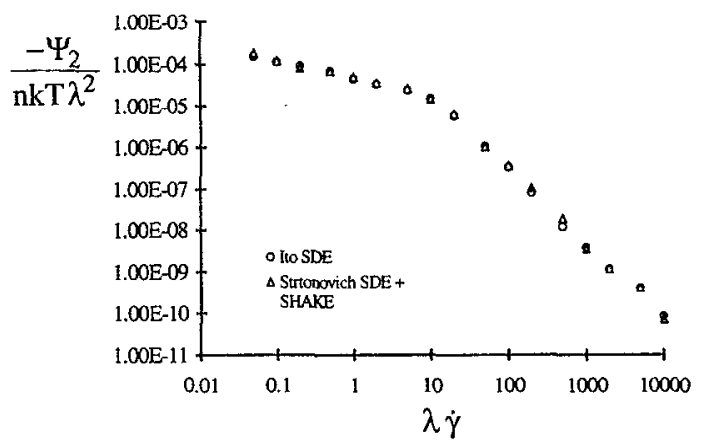

FIG. 3. Comparison of reduced second normal stress difference for rigid dumbbells from Brownian dynamics simulations for Ito SDE (circle), and Stratonovich SDE with SHAKE (triangle).

assume that the argument can be extended to models with an arbitrary number of beads. This is an assumption that should be investigated further in future research.

\section{A. Steady shear flow}

Three characteristic material functions-shear viscosity $\eta$, first normal stress difference $\Psi_{1}$, and second normal stress difference $\Psi_{2}$-are generally measured in steady shear flow. Figure 4 shows the reduced viscosity against shear rate for different link-tension coefficients. Several features of this figure must be discussed. First, the reduced viscosity approaches a plateau at low shear rates $(\lambda \dot{\gamma}<1)$. At low shear rates, the polymer chains are coiled up due to the intramolecular constraint forces and the Brownian forces. We can see that the root-mean-square end-to-end distances remain constant for $\lambda \dot{\gamma}<1$ in Fig. 7. Second, all cases show shearthinning behavior when the shear rate increases. Shearthinning becomes stronger when the link tension coefficient $\epsilon$ becomes small. Note that the component of the difference of hydrodynamic forces on two succeeding beads in the direction of the link becomes small as $\epsilon$ decreases. Since the backbone of the polymer chain tends to align with the flow direction, it means that the resistance will decrease along the shear direction. Third, it appears that the viscosity slowly

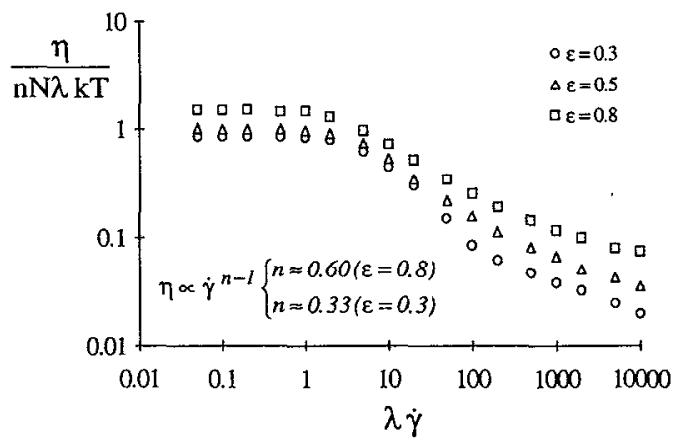

FIG. 4. Reduced steady shear viscosity vs shear rate curves determined from Brownian dynamics simulations of Kramers chains with ten beads and anisotropic hydrodynamic drag [ $\epsilon=0.3$ (circle), $\epsilon=0.5$ (triangle), and $\epsilon=0.8$ (square)]. 


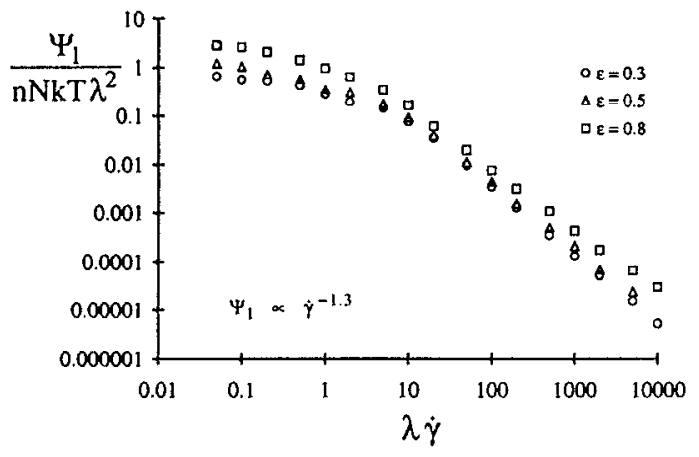

FIG. 5. Reduced first normal stress coefficient vs shear rate curves determined from Brownian dynamics simulations of Kramers chains with ten beads and anisotropic hydrodynamic drag $[\epsilon=0.3$ (circle), $\epsilon=0.5$ (triangle), and $\epsilon=0.8$ (square)].

decreases and does not approach a plateau at high shear rates, as it does in dilute solution. This phenomenon is not in agreement with the Curtiss-Bird model, ${ }^{23}$ in which $\eta^{\circ} \dot{\gamma}^{-1}$, and is different from the prediction for bead-spring models, ${ }^{26}$ which yield a plateau even for shear rates that are well below those studied here.

We also note another interesting fact about the shearthinning bchavior of the viscosities. For all values of $\epsilon$, these follow a "power-law" expression,

$$
\eta \propto \dot{\gamma}^{n-1} \text {. }
$$

The "power-law" parameter $n$ for the bead-rod chain depends on $\epsilon$. When the separation between beads $a$ is held constant, the power-law parameter decreases from $n=3 / 5$ for $\epsilon=0.8$ to $n=1 / 3$ for $\epsilon=0.3$. Experimentally determined values of $n$ range from $0.1-0.6$ for typical polymeric fluids. ${ }^{27}$

Figure 5 shows the first normal stress coefficient $\Psi_{1}$ vs shear rate. The behavior of $\Psi_{1}$ is similar to that of the viscosity function when the shear rate is low. However, there are some differences at high shear rates; $\Psi_{1}$ continues to decrease linearly with shear rate on a $\log -\log$ scale. The slope is near -1.3 , which seems to be consistent with that predicted by the Curtiss-Bird model, in which $\Psi_{1} \propto \dot{\gamma}^{-2} \ln \dot{\gamma}$.

Figure 6 shows the second normal stress coefficient $\Psi_{2}$.

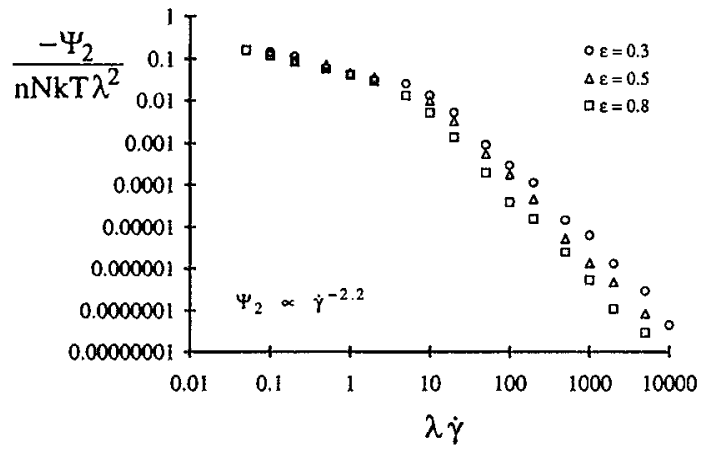

FIG. 6. Reduced second normal stress cocfficient vs shear rate curves determined from Brownian dynamics simulations of Kramers chains with ten beads and anisotropic hydrodynamic drag [ $\epsilon=0.3$ (circle), $\epsilon=0.5$ (triangle), and $\epsilon=0.8$ (square)].

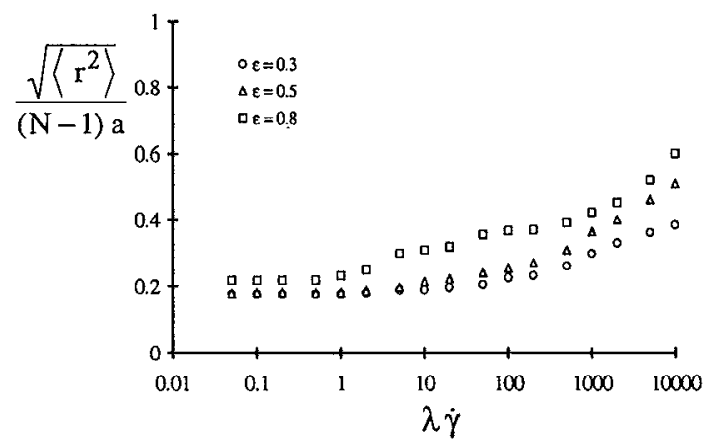

FIG. 7. Mean square root end-to-end distance of Kramers chains with ten beads and anisotropic hydrodynamic drag in steady shear flow determined from Brownian dynamics simulations [ $\epsilon=0.3$ (circle), $\epsilon=0.5$ (triangle), and $\epsilon=0.8$ (square)].

The values of $\Psi_{2}$ are all negative. While the behavior of $\Psi_{2}$ is similar to that of $\Psi_{1}$, Fig. 6 shows that $\Psi_{2}$ decreases much faster as shear rate increases. The slope of $\Psi_{2}$ against $\dot{\gamma}$ on a $\log -\log$ scale is -2.2 , which is also consistent with the value of -2.5 predicted by the Curtiss-Bird model. The simulated zero-shear first and second stress coefficients roughly agree with the relation $\Psi_{2,0} / \Psi_{1,0}=-\frac{2}{7}(1-\epsilon) .{ }^{15}$ However, unlike the viscosity and $\Psi_{1}, \Psi_{2}$ shows higher values for smaller $\epsilon$. Note that $\Psi_{2}=0$ for $\epsilon=1$.

Figure 7 shows the root-mean-square end-to-end distance. At low shear rates, $\sqrt{\left\langle r^{2}\right\rangle}$ is unchanged since the constraint forces and Brownian forces in the model prevent the polymer chain from extending. This is consistent with the nearly constant values for $\eta, \Psi_{1}$, and $\Psi_{2}$ for low shear rates. As the shear rate increases, the end-to-end distance slowly increases. Even at $\lambda \dot{\gamma}=O\left(10^{4}\right)$, we have not reached the asymptotic values expected for high shear rates. When the link tension coefficient $\epsilon$ increases $(\epsilon=0.8)$, the hydrodynamic and Brownian forces become isotropic. These forces lead to a peak in the change of $\sqrt{\left\langle r^{2}\right\rangle}$ vs $\lambda \dot{\gamma} .{ }^{20,21}$ This is why values for $\sqrt{\left\langle r^{2}\right\rangle}$ are slightly shifted upwards in the range of $\lambda \dot{\gamma}$ between 2 and 200. However, such a high value of $\epsilon(=0.8)$ (Ref. 23) is unrealistic for concentrated polymer solutions; we believe that this behavior will not be seen in polymer melts.

Figure 8 shows viscosity functions for 10,20 , and 30 mers with link tension coefficient $\epsilon=0.3$. We can observe

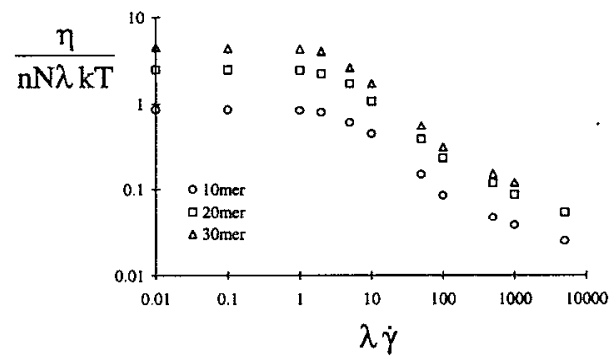

FIG. 8. Reduced steady shear viscosity functions of Kramers chains with 10 (circle), 20 (square), and 30 (triangle) beads determined from Brownian dynamics simulations. 


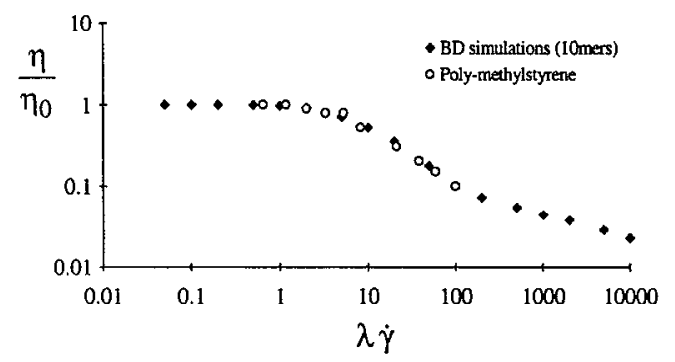

FIG. 9. Simulated steady-state viscosity for 10-mers and experimental data for monodisperse poly- $\alpha$-methylstyrene solution. The data are for $7 \%$ (by weight) solution of poly- $\alpha$-methylstyrene in Kanechlor at $25^{\circ} \mathrm{C}$. The polymer sample has $\bar{M}_{w} / \bar{M}_{n}<1.01$ (Ref. 28 ).

that the effect of chain length is to increase the viscosity. Note, however, that the overall shape of the curves and the onset of shear thinning are not affected by $N$ (for the chains studied here).

Experimental data on $\eta$ and $\Psi_{1}$ for melts and concentrated solutions ${ }^{28,29}$ can be described reasonably well by our model, as may be seen in Figs. 9 (poly- $\alpha$-methylstyrene solutions), 10, and 11 [low density polyethylene (LDPE)]. For all our comparisons to experiments we used $\epsilon=0.3$. Also note that we used the same value of $\lambda$ to fit both the viscosity (Fig. 10) and the first normal stress coefficient (Fig. 11). The agreement for the viscosity of LDPE (Fig. 9) appears to decay for $\lambda \dot{\gamma}>50$. We attribute the disagreement between theory and experiment to the fact that the sample of low density polyethylene used for the experiments was highly polydisperse $\left[\left(\bar{N}_{w} / N_{n}\right)=22\right]$. For monodisperse polymers, agreement is satisfactory over a wide range of shear rates (Fig. 9). We will investigate polydispersity effects in a subsequent paper.

Because of the expense of the computations, to investigate EV effects we limit our attention to the case where $\epsilon-0.5$. Before presenting the results, we fix the parameters appearing in the HS and the soft repulsive potentials. The parameter $C$ and the exponent $m$ determine the strength of the potential [see Eq. (A2)]. We choose $C / k T=4$ for our study. In order to select a value for exponent $m$, we performed simulations for both $m=12$ and $m=20$. The differences for the rheological properties are shown in Table I; these are within the statistical error. We choose $m$ to be 12 .

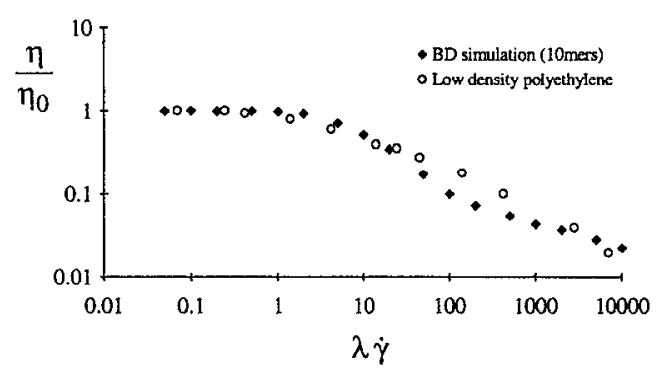

FIG. 10. Simulated steady-state viscosity (for 10-mers) and experimental data for highly polydisperse low density polyethylene at $423 \mathrm{~K}$ (Ref. 29). The polydispersity is approximately 22 .

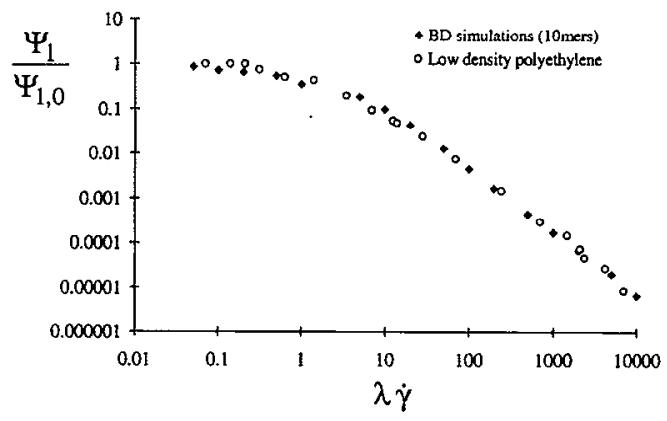

FIG. 11. Simulated first normal stress coefficient (for 10-mers) and experimental data for the same sample shown in Fig. 10 (Ref. 29).

The effects of EV on rheological properties are shown in Figs. 12-15. We also present the results without EV in these figures. Figure 12 shows the viscosity function. The viscosities calculated with a HS or a soft repulsive potential are shifted upwards (with respect to a phantom chain) in the entire range of shear rate studied here. However, there is an important difference between the hard and the soft potentials at high shear rates; the viscosity approaches a second plateau when we consider a hard sphere potential, while it continues to decrease for the phantom chain and for the polymer chain with a soft repulsive potential. We believe that the chain with the soft repulsive potential yields the correct behavior for viscosity, and it shares many similarities with the phantom chain at high shear rates. In addition, the viscosities using a soft repulsive potential are higher than those for a hard sphere potential for $\lambda \dot{\gamma}<500$, at which point there is crossover for the two different potentials. The primary reason for the different viscosities between the HS and the soft repulsive potentials can be traced back to different chain kinematics and an additional contribution from the soft EV forces to the stress tensor. The different chain kinematics can be explained by a different end-to-end distance for the chains. As illustrated in Fig. 15, the longer end-to-end distance will produce a larger resistance to the flow under the same external force, thereby yielding a higher viscosity.

Figures 13 and 14 show the first and second normal stress coefficients, respectively. Unlike the viscosities at low shear rates, the constant values of $\Psi_{1}$ and $\Psi_{2}$ observed with a soft repulsive potential are lower than those observed with a hard sphere potential, As the shear rate increases $(\lambda \dot{\gamma}>10)$, the difference in $\Psi_{1}$ for the two different potentials is within the statistical error, but the values of $\Psi_{2}$ for the soft repulsive potential are slightly below those for the HS potential.

In contrast to our findings in the absence of EV effects, $\Psi_{1}$ decreases slowly with shear rate $\left(\Psi_{1} \propto \dot{\gamma}^{-1.2}\right) ; \Psi_{2}$ decreases much slower than in the absence of $\mathrm{EV}$ forces (the

TABLE I. The differences for the rheological properties.

\begin{tabular}{llccc}
\hline \hline Function & $\eta / n k T \lambda$ & $\Psi_{1} / n k T \lambda^{2}$ & $-\Psi_{2} / n k T \lambda^{2}$ & End-to-end distance \\
\hline$m=12$ & 0.5712 & $2.89 \times 10^{-2}$ & $1.8 \times 10^{-3}$ & 0.43 \\
$m=20$ & 0.547 & $2.74 \times 10^{-2}$ & $1.61 \times 10^{-3}$ & 0.42 \\
rel. err. & $4.24 \%$ & $5.2 \%$ & $10.6 \%$ & $2.3 \%$ \\
\hline \hline
\end{tabular}




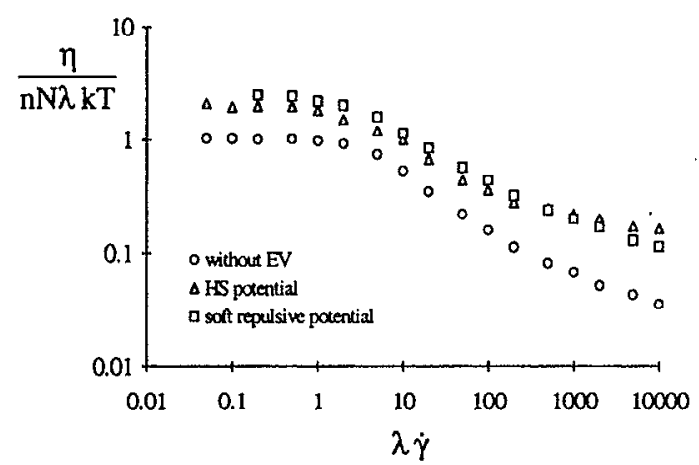

FIG. 12. Excluded volume effects on reduced viscosity functions for Kramers chains with ten beads. The circle shows results for phantom chains, the triangles for a HS potential, and the squares for a soft repulsive potential.

slope is -1.44 when considering EV forces, and -2.2 without EV forces). A detailed interpretation of these differences is postponed to a later section.

Figure 15 shows the behavior of the end-to-end distance with and without EV forces. The end-to-end distance roughly captures the kinematics of the polymer chains. The three curves shown in Fig. 15 indicate that $\sqrt{\left\langle r^{2}\right\rangle}$ approaches a plateau at low shear rates $(\lambda \dot{\gamma}<1)$ and increases monotonically with shear rate. Note, however, that the deformation of the polymer chain in the presence of EV forces is more pronounced than in the absence of EV forces. Furthermore, we notice that $\sqrt{\left\langle r^{2}\right\rangle}$ exhibits a higher value for a soft repulsive potential than for the corresponding HS potential. Since the polymer chain is stretched out after $\lambda \dot{\gamma}>500, \mathrm{EV}$ forces have only a minor effect on chain kinematics at high shear rates. For the bead-rod model under consideration, EV forces lead to somewhat stronger constraint forces (which keep the distance between successive beads constant). The differences in $\sqrt{\left\langle r^{2}\right\rangle}$ due to the two different potential-energy functions employed here (HS and a soft repulsive potential) essentially disappear for high $\lambda \dot{\gamma}$. This implies that the decrease in viscosity after the crossover point (Fig. 12) is mainly due to an additional contribution of $\mathrm{EV}$ forces to the stress tensor.

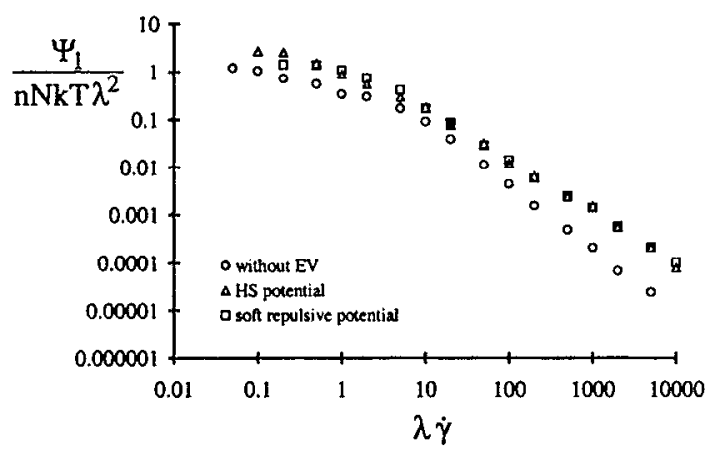

FTG. 13. Excluded volume effects on reduced first normal stress difference for Kramers chains with ten beads. The circles show results for phantom chains, the triangles for chains with a HS potential, and the squares for chains with a soft repulsive potential.

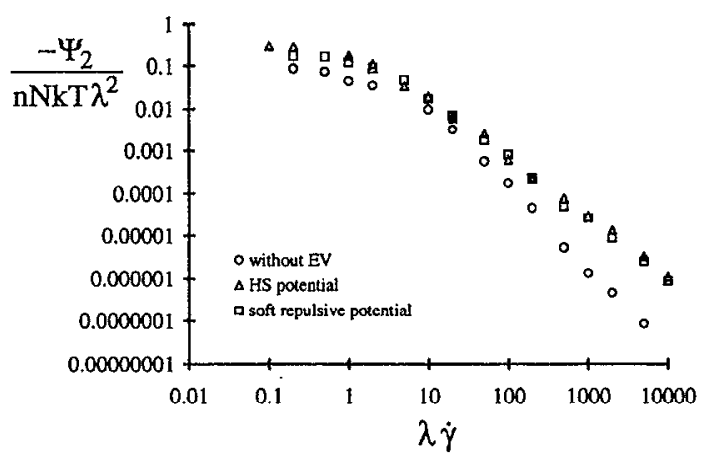

FIG. 14. Excluded volume effects on reduced second normal stress difference for Kramers chains with ten beads. The circles show results for phantom chains, the triangles for chains with a HS potential, and the squares for chains with a soft repulsive potential.

In order to gain further insight into the cffects of EV, we have performed a detailed force analysis for the case with $\lambda \dot{\gamma}=10$. Rather than presenting all the forces acting on every bead, we study the beads at the end, in the middle, and on an average bead inside the chain (which is defined to be an average over beads 2 to 9 in the model). The results can be seen in Figs. 16 and 17 for a soft repulsive potential, Fig. 18 for a hard sphere potential, and Fig. 19 in the absence of EV forces. Only the magnitudes of the forces are shown in these figures, and they have been nondimensionalized by $k T$.

First we analyze hydrodynamic forces (shown in Figs. 16,18 , and 19). Very similar hydrodynamic-force distribution functions are observed for the bead-rod chain with a soft repulsive potential (Fig. 16) and in the absence of EV forces (Fig. 19). The only difference is that the curve of average hydrodynamic force on the inner bead is shifted to the right when $\mathrm{EV}$ forces are not included; the peak of the curve is situated around 750 instead of 700 in the presence of soft repulsive forces. For the bead-rod chain with a HS potential, the peak of average hydrodynamic force on the inner bead is situated at 760 , and the value of the peak is lower than those for a phantom chain and a soft-repulsive-potential chain.

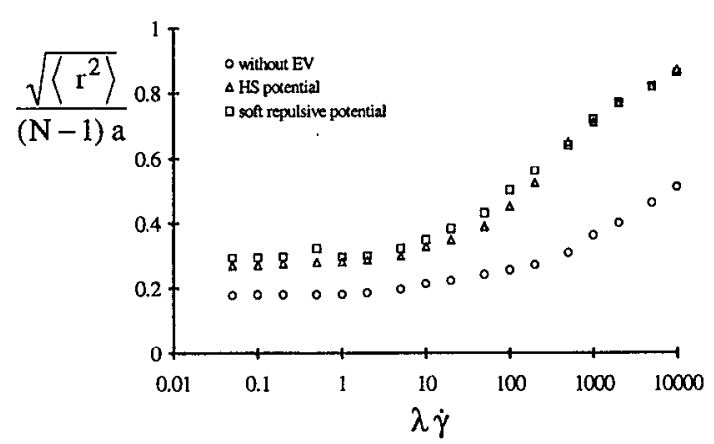

FIG. 15. Excluded volume effects on mean square end-to-end distance for Kramers chains with ten beads. The circles show results for phantom chains, the triangles for chains with a HS potential, and the squares for chains with a soft repulsive potential. 


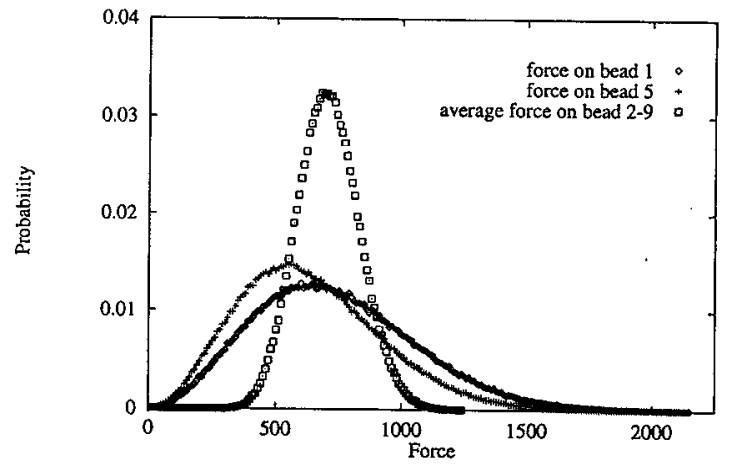

FIG. 16. Distribution functions for the hydrodynamic forces in Kramers chains with ten beads and a soft repulsive potential-energy function. The diamond $(\diamond)$ indicates the force on bead 1 . The plus $(+)$ denotes the force on bead 5 . The square $(\square)$ designates the average force on the inner beads (beads 2-9).

For the soft repulsive potential we computed the force due to EV (shown in Fig. 17). The EV forces are relatively small (in the range of 0-350). The average EV forces acting on the inner beads (bead 2-9) are concentrated in a relatively narrow region ranging from 150 to 300 . For the end beads, a much narrower distribution (almost a delta function) of EV forces is observed. In contrast, the curve corresponding to the middle bead is relatively broad. In order to gain a better understanding of these phenomena, we show in Fig. 20 two simple possible configurations of the polymer chain under external flow fields. This figure illustrates why EV forces on the inner bead are more sensitive to the chain configuration. For instance, when the polymer chain has an " $S$ " shape, the inner bead feels EV forces from both end beads (see Fig. 20). When the configuration of the polymer chain changes to "hairpin," the inner bead feels an EV force from only one end bead (see also Fig. 20). However, the end beads feel roughly the same EV forces for both configurations of the polymer chain. This implies that EV forces on end beads are less sensitive to the configuration of the polymer chain. In addition, the small EV forces suggest that the beads tend to

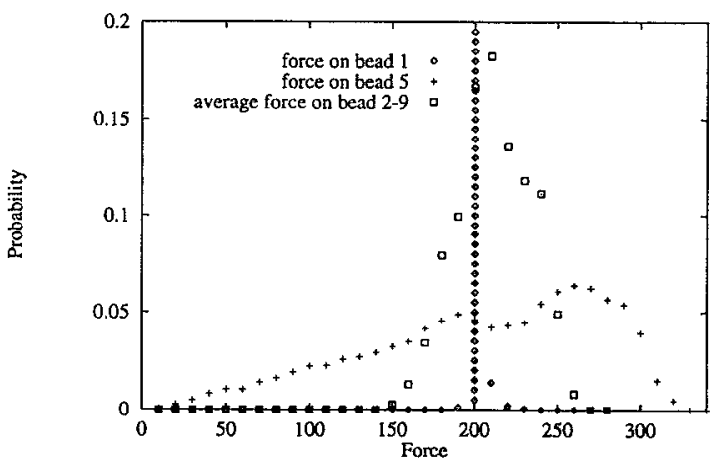

FIG. 17. Distribution functions for EV forces in Kramers chains with ten beads and a soft repulsive potential-energy function. The diamond $(\diamond)$ indicales the force on bead 1 . The plus $(+)$ denotes the force on bead 5 . The square $(\square)$ designates the average force on the inner beads (beads 2-9).

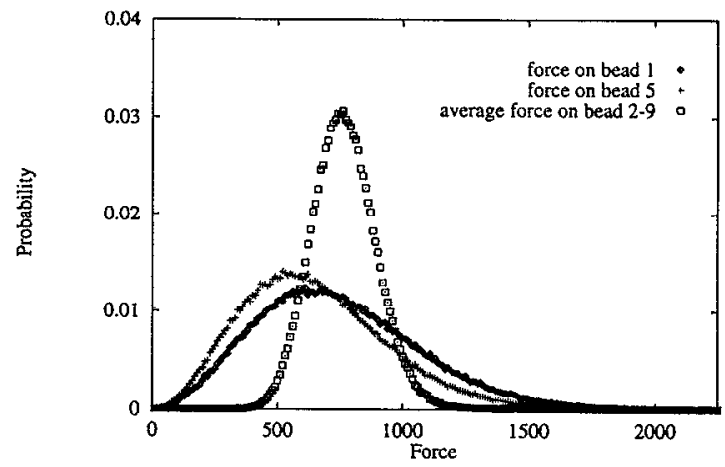

FIG. 18. Distribution functions for the hydrodynamic forces in Kramers chains with ten beads and hard sphere potential-energy function. The diamond $(\diamond)$ indicates the force on bead 1 . The plus $(+)$ denotes the force on bead 5 . The square $(\square)$ designates the average force on the inner beads (beads 2-9).

be separated from each other, thereby leading to trajectories that yield a much larger end-to-end distance $\sqrt{\left\langle r^{2}\right\rangle}$ than a phantom chain.

In the present study, we have observed significant differences in the rheological properties of a polymer melt when two types of repulsive potential-energies are employed. As mentioned earlier, this difference can be traced back to the different chain kinematics and force contributions to the stress tensor. Comparing the distribution function of averaged hydrodynamic force shown in Fig. 16 with that shown in Fig. 18, we can see that not only is the curve with a HS potential shifted to a higher force regime, but also the probability at the peak is lower than that for a soft repulsive potential. We already know that the end-to-end distance between beads for a HS potential is smaller than that for a soft repulsive potential at $\lambda \dot{\gamma}=10$. The combination of these features results in a lower viscosity at $\lambda \dot{\gamma}=10$ for the HS potential. We therefore conclude that ignoring $\mathrm{EV}$ forces in the expression for the stress tensor, but taking them into account indirectly through an algorithm that discards "overlapping" configurations is incorrect. The discrepancy between

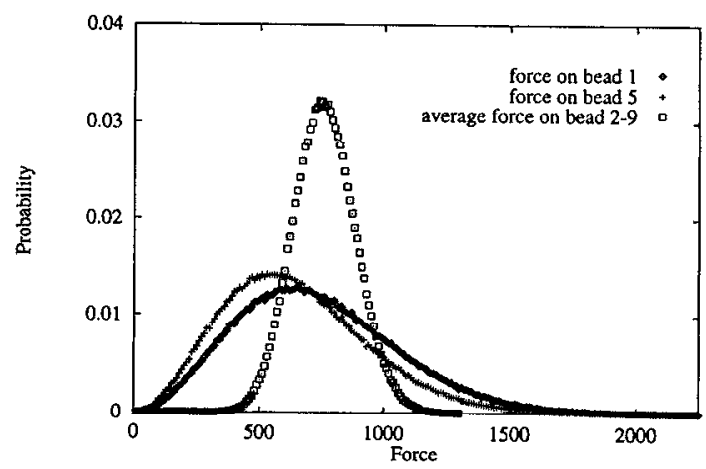

FIG. 19. Distribution functions for the hydrodynamic forces in Kramers chains with ten beads without excluded volume. The diamond $(\diamond)$ indicates the force on bead 1 . The plus $(+)$ denotes the force on bead 5 . The square designates the average force on the inner beads (beads 2-9). 


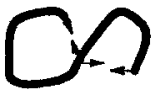

"S" shape

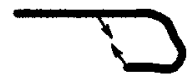

hairpin
FIG. 20. Two representative configurations of the polymer chain.

our results for a HS and a soft repulsive potential show clearly that EV forces must be taken into account in the expression for the stress tensor. Furthermore, we find that a naive hard sphere potential treatment will lead to an incorrect behavior of the viscosity function at high shear rates.

\section{B. Steady elongational flow}

In steady simple elongational flow only one characteristic material function-the elongational viscosity - can be measured. Figure 21 shows elongational viscosities for freely-jointed bead-rod chains with link tension coefficients $\epsilon=0.3,0.5$, and 0.8 . For all three values of $\epsilon$ a transition is observed from low elongational viscosities at low elongational rates to high elongational viscosities at high elongational rates. This transition occurs at lower elongational rates and is less sharp when the link tension coefficient $\epsilon$ increases. We did not observe any evidence for elongational thinning. The corresponding root-mean-square end-to-end distances, shown in Fig. 22, exhibit a behavior similar to that of the elongational viscosities. Polymer chains are subject to a conformational transition in going from low elongational rates to high elongational rates. After the transition, the polymer chain is nearly fully stretched out.

The effects of EV on elongational viscosities can be predicted without need for further simulations. If we take EV forces into account, the values of elongational viscosities would be shifted up at low elongational rates, but high elongational viscosities would remain unchanged because they correspond to nearly fully stretched out chain conformations. The behavior of elongational viscosities when including EV forces will be similar to that shown in Fig. 21.

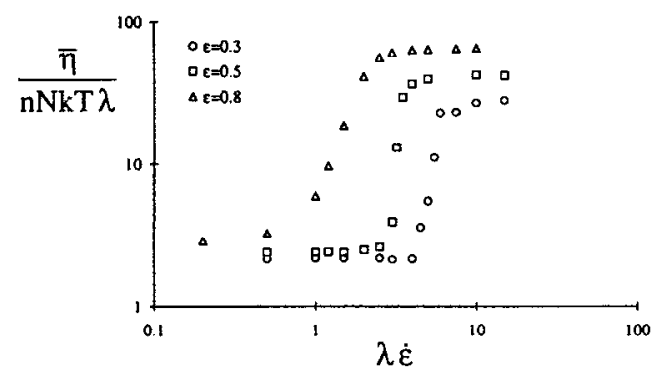

FT. 21. Steady elongational viscosity of Kramers chains with ten beads and anisotropic hydrodynamic drag determined from Brownian dynamics simulations for three different link tension coefficients [ $\epsilon=0.3$ (circle), $\epsilon=0.5$ (square), and $\epsilon=0.8$ (triangle)].

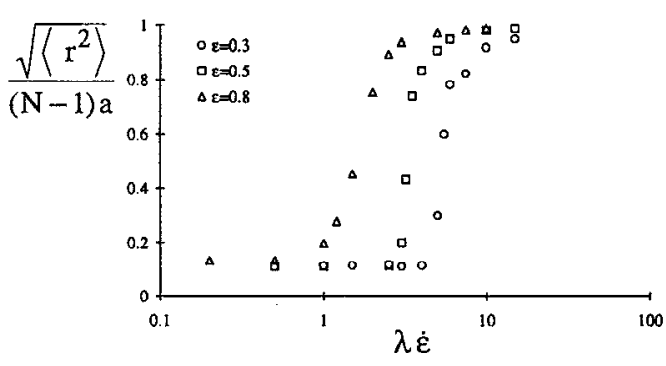

FIG. 22. Mean square root end-to-end distance of Kramers chains with ten beads and anisotropic hydrodynamic drag in steady simple elongational flow determined from Brownian dynamics simulations for three different link tension coefficients [ $\epsilon=0.3$ (circle), $\epsilon=0.5$ (square), and $\epsilon=0.8$ (trianglc)].

\section{CONCLUSION}

The anisotropic hydrodynamic forces introduced in this work have permitted the study of a fairly well resolved mean field model for concentrated polymer solutions. The anisotropy is assumed to hinder the motion of each link in the freely-jointed bead-rod polymer chain in a direction perpendicular to the backbone and hence depends on the actual configuration vector. The corresponding anisotropic Brownian forces satisfy the fluctuation-dissipation theorem. We have derived the Fokker-Planck equation for the model from a set of Stratonovich stochastic differential equations; since its analytical solution is difficult, we have conducted Brownian dynamics simulations based on the set of Stratonovich SDEs combined with the SHAKE algorithm. For dumbbells, the trajectories constructed by this approach have been shown to converge to those from the equivalent Ito SDE.

Nonlinear rheological properties, such as viscosity, and first and second normal stress differences in steady shear flow have been calculated for different link tension coefficients. All curves are in qualitative agreement with experiment and other available simulations.

As expected, we find that excluded volume has a strong effect on the rheological properties and chain kinematics, particularly for short chains. We also find that a naive algorithm to incorporate hard sphere interactions into our simulations leads to incorrect results for the viscosity at high shear rates; a "proper" way of taking excluded volume into account is through a soft repulsive potential and an extrapolation of the results to the case of an infinitely "stiff" potential.

The most severe approximation made in this work is perhaps Eq. (10) for the total hydrodynamic force acting on a macromolecule. A possible improvement of this approximation could be to assume the form of the hydrodynamic force acting on the end bead instead of a total hydrodynamic force on a molecule in order to close Eq. (19). Behind this latter assumption lies the concept of the reptating motion of a polymer chain in a concentrated solution. When it reptates, a polymer chain moves along its backbone and the bead at the end of the chain experiences a different hydrodynamic drag than the inner beads; end beads actually move more freely than inner beads. The consequences of such an assumption, however, need to be further investigated. 
We should also point out that while the freely-jointed bead-rod chain has some theoretical advantages over the bead-spring chain, ${ }^{15}$ its mathematical treatment makes it unattractive from a computational point of view. The convergence of the SHAKE algorithm that we have adopted in this work deteriorates for long chains and requires extensive calculations.

\section{ACKNOWLEDGMENTS}

This material is based on projects supported by the National Science Foundation (CTS). J.D.P. is grateful to the Camille and Henry Dreyfus Foundation for a New Faculty Award. We are indebted to Professor R. B. Bird for valuable discussions and helpful comments. We gratefully acknowledge the use of the CONDOR network of $100+$ DECStations in the UW-Madison Computer Science Department. We are also grateful to the Pittsburg Supercomputing Center, where some of our calculations have been performed.

\section{APPENDIX A: SDE WITH A SOFT REPULSIVE POTENTIAL}

The stochastic differential equations for the model including a soft repulsive potential-energy function are

$$
\begin{aligned}
\dot{\mathbf{r}}_{\nu}= & \kappa \cdot \mathbf{r}_{\nu}+\alpha \sum_{\mu} \mathbf{H}_{\nu \mu} \cdot \mathbf{f}_{\mu}+\sum_{\mu} \tilde{\zeta}_{\nu \mu}^{-1} \cdot \mathbf{F}_{\mu}^{(c)}+\sum_{\mu} \tilde{\zeta}_{\nu \mu}^{-1} \\
& \cdot \mathbf{F}_{\mu}^{(\mathrm{eV})} .
\end{aligned}
$$

Here $\mathbf{F}_{\mu}^{(\mathrm{eV})}$ denotes the excluded volume force acting on bead $\mu$ due to the interaction with all other beads of the chain. By modelling the interaction as a soft repulsive potential-energy, we can obtain

$$
\mathbf{F}_{\mu}^{(\mathrm{eV})}=4 m \frac{C}{k T} \sum_{\eta}\left(\frac{d}{r_{\mu \eta}}\right)^{m} \frac{\mathbf{r}_{\mu \eta}}{\mathbf{r}_{\mu \eta}^{2}} .
$$

Here $r_{\mu \eta}$ is the distance between two beads $\mu$ and $\eta$. The parameter $d$ denotes a characteristic length scale for the separation between beads. $C$ is a parameter with units of energy, $k$ is Boltzmann's constant, and $T$ is the bath's temperature. When exponent $m$ is equal to 12 , the interaction force corresponds to the repulsive part of a $\mathrm{LJ}$ potential energy function.

${ }^{1}$ P. G. de Gennes, J. Chem. Phys. 55, 572 (1971).

${ }^{2}$ P. G. de Gennes, Scaling Concepts in Polymer Physics (Cornell University, Ithaca, 1979).

${ }^{3}$ M. Doi and S. F. Edwards, J. Chem. Soc. Faraday Trans. 74, 1789 (1978).

${ }^{4}$ M. Doi and S. F. Edwards, J. Chem. Soc. Faraday Trans. 74, 1802 (1978).

${ }^{5}$ M. Doi and S. F. Edwards, J. Chem. Soc. Faraday Trans. 74, 1818 (1978).

${ }^{6} \mathrm{M}$. Doi and S. F. Edwards, J. Chem. Soc. Faraday Trans. 75, 38 (1979).

${ }^{7} \mathrm{M}$. Doi and S. F. Edwards, The Theory of Polymer Dynamics (Clarendon, Oxford, 1986).

${ }^{8}$ C. F. Curtiss and R. B. Bird, J. Chem. Phys. 74, 2016 (1981).

${ }^{9}$ C. F. Curtiss and R. B. Bird, J. Chem. Phys. 74, 2025 (1981).

${ }^{10}$ R. B. Bird and J. R. DeAguiar, J. Non-Newtonian Fluid Mech. 13, 149 (1983).

${ }^{11}$ J. R. DeAguiar, J. Non-Newtonian Fluid Mech. 13, 161 (1983).

${ }^{12}$ X. J. Fan, J. Non-Newtonian Fluid Mech. 17, 251 (1985).

${ }^{13}$ V. S. Volkov and G. V. Vinogradov, Rheol. Acta 23, 231 (1984).

${ }^{14} \mathrm{~N}$. Phan-Thien and J. D. Atkinson, J. Non-Newtonian Fluid Mech. 17, 111 (1985).

${ }^{15}$ R. B. Bird, C. F. Curtiss, R. C. Armstrong, and O. Hassager, Dynamics of Polymeric Liquids, 2nd ed. (Wiley-Interscience, New York, 1987), Vol. 2.

${ }^{16}$ L. G. Baxandall, J. Chem. Phys. 87, 2297 (1987).

${ }^{17}$ R. S. Parnas and Y. Cohen, J. Chem. Phys. 90, 6680 (1989).

${ }^{18}$ M. Fixman, J. Chem. Phys. 45, 785 (1966).

${ }^{19}$ K. H. Ahn and S. J. Lee, J. Non-Newtonian Fluid Mech. 43, 143 (1992).

${ }^{20}$ T. W. Liu, J. Chem. Phys. 90, 5826 (1989).

${ }^{21}$ T. W. Liu, Ph.D. thesis, University of Wisconsin-Madison, 1989.

${ }^{22}$ R. B. Bird, H. H. Saab, and C. F. Curtiss, J. Chem. Phys. 77, 4747 (1982).

${ }^{23}$ H. H. Saab, R. B. Bird, and C. F. Curtiss, J. Chem. Phys. 77, 4758 (1982).

${ }^{24}$ J. D. Schieber and H. C. Ottinger, J. Chem. Phys. 89, 6972 (1988).

${ }^{25}$ J. P. Ryckaert, G. Ciccotti, and H. J. C. Berendsen, J. Comput. Phys. 23, 327 (1977).

${ }^{26} \mathrm{P}$. Biller and F. Petruccione, J. Chem. Phys. 89, 2412 (1988).

${ }^{27}$ R. B. Bird, R. C. Armstrong, and O. Hassager, Dynamics of Polymeric Liquids, 2nd ed. (Wiley-Interscience, New York, 1987), Vol. 1.

${ }^{28} \mathrm{H}$. Endo, T. Fujimoto, and M. Nagasawa, J. Polym. Sci. A 2, 345 (1970). ${ }^{29}$ H. M. Laun, J. Rheol. 30, 459 (1986). 